\title{
AUTOCONHECIMENTO: UMA BREVE REVISÃO NARRATIVA
}

\author{
Maria João Carapeto \\ Universidade de Évora, Escola de Ciências Sociais, Departamento de Psicologia \\ mjoaocarapeto@gmail.com
}

Recepción Artículo: 27 octubre 2021 Admisión Evaluación: 27 octubre 2021 Informe Evaluador 1: 28 octubre 2021 Informe Evaluador 2: 29 octubre 2021 Aprobación Publicación: 30 octubre 2021

\section{RESUMO}

No final do século XIX, William James escreveu um dos primeiros e mais inspiradores documentos sobre 0 autoconhecimento da história da psicologia moderna. No entanto, só meados do século XX a literatura do autoconceito coloca definitivamente 0 autoconhecimento como tema de investigação científica sistemática. Desde então, a investigação não tem parado e, especialmente dos anos 80 do século XX em diante, importantes contributos têm vindo abrir novas direções até à atualidade. Apresenta-se aqui uma revisão narrativa não exaustiva de alguns desses contributos e novas direções. Entre outros, aborda-se a multiplicidade e a diversidade das representações que construímos sobre nós próprios; a sua organização estrutural, da estrutura como forma estática e reificada às concetualizações mais recentes de uma estrutura dinâmica; a estabilidade do autoconhecimento mas também a sua variabilidade; ou a relação do autoconhecimento com a adaptação ou as suas funções adaptativas; e uma nota sobre alguns dos contributos recentes das neurociências cognitivas. Em conclusão, a investigação e as teorias do autoconhecimento têm vindo a esboçar trajetórias de conciliação com a complexidade da experiência de si mesmo de cada indivíduo e com a riqueza conceptual dos primeiros autores, muito se devendo aos avanços também registados ao nível das metodologias de investigação e análise de dados.

Palavras-chave: autoconceito; autoestima; identidade; desenvolvimento; estrutura do autoconhecimento; adaptação

\section{ABSTRACT}

Self-knowledge: a brief narrative review. In the late nineteenth century, William James wrote one of the first and most inspiring documents on self-knowledge in the history of modern psychology. However, it was not until the mid-twentieth century that the literature on self-concept definitively placed self-knowledge as a subject for systematic scientific research. Since then, research has not stopped and, especially from the 80s of the 20th century onwards, important contributions have been offering new directions until today. A non-exhaustive narrative review of some of these contributions and new directions is presented here. Among others, it addresses 


\section{AUTOCONHECIMENTO: UMA BREVE REVISÃO NARRATIVA}

the multiplicity and diversity of the representations we build about ourselves; its structural organization, from a static and reified structure to the most recent conceptualizations of a dynamic structure; the stability of self-knowledge but also its variability; or the relationship of self-knowledge to adaptation, or its adaptive functions; and a note on some recent contributions of cognitive neurosciences. In conclusion, research and theories of self-knowledge have been building paths of reconciliation with the complexity of each individual's experience of himself and the conceptual richness of the first authors, largely due to the advances also registered in the fields of research methodologies and data analysis.

Keywords: self-concept; self-esteem; identity; development; structure of self-knowledge; adaptation

\section{INTRODUÇÃO}

0 autoconhecimento é um tema central da psicologia moderna (James, 1980). Contudo, só em meados do século XX a literatura do autoconceito o coloca definitivamente como tema de investigação científica sistemática, conquistando também um reconhecimento que se mantem até aos dias de hoje (Bracken, 1996; Byrne, 2002; Harter, 2012). Não obstante a posição central que continua a ter e os avanços teóricos e metodológicos decorridos, muitas limitações têm sido apontadas e um conjunto de novas direções tem vindo a consolidar-se. Faz-se aqui precisamente uma revisão narrativa, não exaustiva, de algumas dessas novas tendências, em contraponto com algumas das caraterísticas da abordagem tradicional do autoconceito.

\section{A ABORDAGEM TRADICIONAL DO AUTOCONCEITO}

Meados do século XX, a revolução cognitiva aliada ao objetivismo reinante, abriu caminho ao estudo sistemático do autoconhecimento. 0 contexto sociocultural da época nas sociedades ocidentais (sobretudo nos Estados Unidos da América), especialmente a crença na singularidade de cada indivíduo, na existência de um "eu" uno, coerente, estável e duradouro, de alguma forma escondido no íntimo de cada pessoa e, por outro lado, os valores da autoexploração, favoreceram a investigação de um autoconhecimento observável, objetivo e mensurável (Baumeister, 1987; Gergen, 1991).

Surgem assim os construtos de "autoconceito", referindo-se essencialmente às descrições que a pessoa faz de si própria, e de "autoestima", dando conta duma dimensão avaliativa ou afetiva da pessoa para consigo mesma, construtos frequentemente tratados de modo indistinto. Construíram-se instrumentos para os medir, em geral escalas que apresentam um número de autodescrições, generalizadas e descontextualizadas (e.g., "sou capaz de fazer as coisas tão bem quanto a maior parte das outras pessoas"; M. Rosenberg, 1965) relativamente às quais a pessoa deve indicar a medida em que nelas se reconhece (Byrne, 2002). No essencial, a primeira geração de instrumentos propunha um autoconceito global, unidimensional, estável, variando quantitativamente de pessoa para pessoa, de tal forma que umas pessoas teriam um autoconceito mais elevado do que outras. Muitos reparos foram feitos à natureza "ateórica" desta investigação e às suas limitações conceptuais (Baumeister et al., 2003; Byrne, 2002). Preocupado com o rumo desta linha de investigação, em 1955, Allport dizia "I greatly fear that the lazy tendency to employ self or ego as a factotum to repair the ravages of positivism may do more harm than good" (p. 38).

Nos anos 1970, novas linhas de investigação abrem caminho a uma nova compreensão do autoconceito (e.g., Epstein, 1973; Markus, 1977), que ganha força nas décadas seguintes.

\section{A MULTIPLICIDADE DO AUTOCONHECIMENTO}

A investigação tem vindo a mostrar que as pessoas constroem uma diversidade de representações de si mesmas, muito para além das autodescrições generalizadas do autoconceito unidimensional. Por exemplo, verificouse que as pessoas se descrevem de modo diferente em diferentes contextos relacionais (e.g., com a mãe e com um amigo) ou em diferentes papéis psicossociais (como trabalhador, como pai, etc.) (Chen et al., 2011; Gergen, 1991; Harter, 2012). Os próprios modelos unidimensionais do autoconceito deram lugar a modelos multidimensionais, mostrando que as pessoas se descrevem de modo diferente em diferentes domínios da sua vida (aparên- 
cia física, relações interpessoais, competências escolares, etc.) (Bracken, 1996; Byrne, 2002). Revelou-se também que as pessoas elaboram representações de si mesmas não só no tempo presente, mas também no passado ou em estados futuros ("eus possíveis"; Markus \& Nurius, 1986), incluindo "eus ideais", "eus devidos", "eus temidos" ou "eus esperados" (Higgins, 1987; Oosterwegel \& Oppenheimer, 1993).

Algumas autorrepresentações envolvem diretamente os outros. É 0 caso das representações do ponto de vista de outros sobre 0 eu (e.g., "o meu amigo acha que sou indiscreta") (Hermans, 2018; Higgins, 1987; James, 1890; Oosterwegel \& Oppenheimer, 1993), ou da ligação entre o conhecimento que a pessoa constrói acerca de si mesma e acerca dos outros (Chen et al., 2011; Kelly, 1955; S. Rosenberg, 1997). Uma pessoa usa as mesmas dimensões de significado (e.g., responsável vs. irresponsável) para compreender os outros e para definir-se a si mesma (Kelly, 1955). Ou, os indivíduos incluem no "eu" as identidades, recursos e perspetivas dos outros com quem têm relações de intimidade, e tratam-nos como se pessoais fossem (Aron \& McLaughlin-Volpe, 2001).

Alguns autores propõem ainda que 0 autoconhecimento extravasa os limites físicos da pessoa e deixa marcas naqueles (e naquilo) com que interage ("eu distribuído") (Kashima et al., 2001). Esta conceptualização parece especialmente promissora para a compreensão das possíveis implicações do uso das novas tecnologias (e.g., redes sociais na internet) na construção do autoconhecimento (Gardner \& Davis, 2013).

\section{A ORGANIZAÇÃO DO AUTOCONHECIMENTO}

0 interesse pelo estudo da organização ou estrutura do autoconhecimento procura responder à questão de como convive tal diversidade de autoconhecimentos no espaço psicológico da pessoa. Alguns autores procuram esclarecer quão longe vai a pluralidade, ou diferenciação, das facetas pessoais distintas que uma pessoa pode construir, e o tipo de ligação entre essas diferentes facetas, ou integração (Bracken, 1996; Campbell et al., 2003; Higgins, 1987; Kelly, 1955; Linville, 1987; Showers \& Zeigler-Hill, 2007).

Embora seja relativamente consensual que 0 autoconhecimento é, de algum modo, diferenciado, existem divergências sobre se essa diversidade é integrada e como. A integração hierárquica tem sido a mais frequentemente defendida (Fischer \& Bidell, 2006; Kelly, 1955; S. Rosenberg, 1997; Shavelson et al., 1976), colocando 0 autoconceito global ou autoestima (Shavelson et al., 1976) ou algum tema nuclear da identidade (Kelly, 1955) no topo da hierarquia. Têm sido também propostas a integração avaliativa, ou medida em que a autodescrição num determinado papel ou contexto social inclui tanto caraterísticas positivas como negativas (Showers \& Zeigler-Hill, 2007) ou a possibilidade da valência (positiva ou negativa) de uns atributos influenciar ou contagiar a valência de atributos vizinhos (Nowak et al., 2005), e a integração narrativa, esta propondo que a coerência global do autossistema é construída por meios autobiográficos e narrativos (Gergen, 1991; Hermans, 2018; McAdams \& McLean, 2013).

Outros temas de interesse têm sido a diferenciação de aspetos específicos do autoconhecimento, como as discrepâncias "eu real"-"eu ideal" (Carapeto \& Feixas, 2020; Higgins, 1987) ou "eu real"-"outros" (Carapeto \& Feixas, 2020; Van Doeselaar et al., 2018), ou os conflitos no seio do autoconhecimento (Carapeto \& Feixas, 2020; Harter, 2012; Oosterwegel \& Oppenheimer, 1993).

De modo geral, a investigação sobre a organização do autoconhecimento tem vindo a distanciar-se do paradigma da estrutura como forma estática, coisificada, garante da previsibilidade do comportamento, ao mesmo tempo que se tem vindo a reconhecer a natureza dinâmica e variável do autoconhecimento e sua organização (Fischer \& Bidell, 2006; Harter, 2012; Nowak et al., 2005; Rafaeli-Mor \& Steinberg, 2002; Showers \& Zeigler-Hill, 2007).

\section{VARIABILIDADE VS. ESTABILIDADE DO AUTOCONHECIMENTO}

Ao focar dimensões mais estáveis do autoconhecimento, o modelo tradicional do autoconceito terá deixando por explicar a sua variabilidade, que a experiência subjetiva das pessoas e a investigação também evidenciam. A perspetiva desenvolvimentista interessa-se pelas mudanças dos conteúdos e da estrutura do autoconhecimento 


\section{AUTOCONHECIMENTO: UMA BREVE REVISÃO NARRATIVA}

ao longo do ciclo de vida, especialmente as normativas. Harter (2012), por exemplo, propôs que as habilidades cognitivas crescentes combinadas com novas expetativas sociais a cada etapa da vida, impulsionam mudanças (possíveis e necessárias), de sofisticação crescente, no autoconhecimento. Tem também vindo a crescer o interesse pelo estudo do microdesenvolvimento (Fischer \& Bidell, 2006), ou das mudanças mais graduais do autoconhecimento, à escala de minutos, horas ou dias, à medida que se avança na realização de uma tarefa (Lichtwarck-Aschoff et al., 2008).

É também possível estudar a variabilidade trans-situacional do autoconhecimento. Situações percebidas como diferentes ativam diferentes configurações de autoconhecimento, e estas configurações mudam dinamicamente com 0 evoluir da situação (Chen et al., 2011; Gergen, 1991; Harter, 2012; Hermans, 2018; Markus \& Kunda, 1986). Markus e Nurius (1986) proposeram que, dependendo da estimulação que a pessoa recebe, só uma pequena parte do autoconhecimento está ativa em cada momento, o "autoconceito operativo". Hermans (2018) propõe o construto de "eu dialógico" para dar conta do diálogo interno permanente que propõe existir entre diferentes representações de si mesmo (e de outros). Higgins (1987) distingue diferentes níveis de acessibilidade das autorrepresentações, umas mais imediatamente disponíveis para o processamento da informação autorreferente do que outras. Outros estudos sugerem que, em determinadas situações, como perante um acontecimento stressante, 0 autoconhecimento se reorganiza de modo particular e transitório (Harter, 2012; Kelly, 1955; Nowak et al., 2005; Showers \& Zigler-Hill, 2007).

Esta atenção à variabilidade apela ao paradigma do estruturalismo dinâmico (Fischer \& Bidell, 2006). À luz deste novo paradigma, é possível observar ambas, estabilidade e variabilidade, desde que sejam usadas diferentes metodologias de investigação, se aceda a diferentes tipos de autoconhecimento ou atenda a diferentes escalas temporais (Bosma \& Kunnen, 2001; Fischer \& Bidell, 2006). As reorganizações descritas como variabilidade podem ser vistas como perturbações (provavelmente transitórias) do autossistema por efeito de algum acontecimento externo ou interno, remetendo para um autoconhecimento "barométrico" (M. Rosenberg, 1986). A estabilidade pode ser entendida como o padrão a que o autossistema regressa quando deixado em repouso, isto é, quando não perturbado por estimulação interna ou externa demasiado exigente do ponto de vista da acomodação (Nowak et al., 2005). Os conceitos de atrator (da teoria dos sistemas dinâmicos) (e.g., Bosma \& Kunnen, 2001; Fischer \& Bidell, 2006; Nowak et al., 2005) e de autoconhecimento "baseline" (M. Rosenberg, 1986) permitem compreender esta estabilidade.

\section{AUTOCONHECIMENTO E ADAPTAÇÃO}

A perspetiva tradicional afirmou a importância do autoconceito para o ajustamento psicológico. Todavia, tem sido criticada pela falta de modelos teóricos que expliquem essa relação e, por outro lado, porque os resultados da investigação nem sempre a têm apoiado (Baumeister et al., 2003; van der Meulen, 2001). A metáfora do autoconhecimento como teoria pessoal, por outro lado, tem sido promissora. Epstein (1973), um dos seus primeiros defensores, definiu "the self-concept ... is a theory that the individual has unwittingly constructed about himself as an experiencing, functional individual" (p. 407). Nesta conceptualização, o autoconhecimento permite ao indivíduo antecipar e interpretar os acontecimentos do seu mundo, guiar a atuação pessoal e otimizar o bem-estar (Harter, 2012; Higgins, 1996; Kelly, 1955; Markus \& Nurius, 1986; McAdams \& McLean, 2013). A metáfora do autorresumo ("self-digest") de Higgins (1996) ilustra também as funções adaptativas do autoconhecimento:

a noção de autorresumo procura captar a ideia de que 0 auto-conhecimento sumariza informação acerca do próprio como um objecto no mundo com o objectivo de servir funções auto-regulatórias. 0 auto-resumo representa o conhecimento acerca de si próprio como um objecto no mundo porque, e na medida em que, esse conhecimento facilita a adaptação ao meio ambiente. 0 auto-resumo é um instrumento para a sobrevivência (p.1063).

No geral, a estimulação ambiental e interna determina que autoconhecimento fica operativo e vai orientar 0 comportamento pessoal (Markus \& Nurius, 1986). Além disso, defende-se que 0 autoconhecimento e a sua mudança desenvolvimental servem a adaptação da pessoa aos ambientes (especialmente os sociais) em que vive. 
Harter (2012), por exemplo, considera que 0 autoconhecimento muda, em boa parte, porque a pessoa necessita de novos guiões para a sua atuação face aos desafios (tarefas de desenvolvimento, acontecimentos de vida) que os ambientes sociais Ihe colocam e um autoconhecimento mais diferenciado e hierarquicamente integrado possibilita uma maior adaptabilidade (Harter, 2012).

De modo diferente, a perspetiva sociocultural propõe que cada cultura promove nos seus membros um modelo particular de "eu" ("self-construal"), procurando assim maximizar o ajustamento dos indivíduos às expectativas sociais que sobre eles recaem no presente e no futuro (Gergen, 1991; Markus \& Kitayama, 2010). Têm sido distinguidos dois modelos de "eu", independente e interdependente, promovidos respetivamente em culturas individualistas e coletivistas (Markus \& Kitayama, 2010). Por outro lado, tem sido sublinhado o valor adaptativo dum modelo de múltiplos "eus relacionais" nas sociedades pós-modernas, caraterizadas pela multiplicidade e variabilidade das relações num mundo global marcado por mudanças tecnológicas rápidas (Cross et al., 2000; Gergen, 1991).

Autoconhecimento e adaptação têm sido relacionados também através da experiência emocional/afetiva (Damásio, 2010; Kelly, 1955; Lazarus, 1991). Van der Meulen (2001) distinguiu três tipos de relação entre autoconhecimento e emoções: (1) a consciência de si próprio é um requisito para a experiência emocional; (2) as emoções constituem indicadores de consciência de si próprio e dos atos pessoais, especialmente as emoções autoconscientes (e.g., vergonha, orgulho); (3) as emoções podem ser vistas como dimensões do autoconceito; (4) a autoestima é frequentemente vista como a componente afetiva do autoconceito; (5) é possível estabelecer relações causais entre autoconceito e emoções (e.g., sentir tristeza quando se descobre não possuir certa característica pessoalmente importante); (6) em vários modelos do autoconhecimento, emoções e cognições aparecem entrelaçadas (e.g., experiências emocionais negativas assinalam a necessidade do autossistema ser revisto; Kelly, 1955).

Tanto os conteúdos como a organização do autoconhecimento têm sido relacionados com a experiência emocional. Por exemplo, a elevada frequência de atributos negativos autopercebidos é caraterística da depressão (Beck et al., 1979; Kelly, 1955) e a sua centralidade tem sido observada em crianças maltratadas (Fischer \& Ayoub, 1994).

Por outro lado, o papel da diferenciação e da integração do autoconhecimento na experiência emocional tem sido um tema controverso. Alguns estudos sugerem que uma maior diferenciação pode amortecer as reações depressivas a acontecimentos adversos (Rafaeli-Mor \& Steinberg, 2002; Showers \& Zeigler-Hill, 2007). Mas tem sido também defendida a importância da integração e coerência do autoconhecimento, dados o sentimento pessoal prazeroso de continuidade temporal e trans-situacional experienciado pela maioria das pessoas, ou o surgir de perturbações dissociativas ou conflitos psicológicos dolorosamente vividos, quando (e enquanto) essa integração não acontece (Campbell et al., 2003; Erikson, 1959; Harter, 2012; Higgins, 1987, 1996; McAdams \& McLean, 2013; Oosterwegel \& Oppenheimer, 1993).

Também os conflitos no autoconhecimento têm sido associados a alterações afetivas (Carapeto \& Feixas, 2019; Harter, 2012; Higgins, 1987), tal como as discrepâncias entre o "eu real" e o "eu ideal" (Carapeto \& Feixas, 2019; Higgins, 1987; Mason et al., 2019). Simultaneamente, aos mesmos conflitos e autodiscrepâncias, tem sido atribuído também um papel positivo na motivação para a ação e na mudança adaptativa (e.g., Higgins, 1996; Markus \& Nurius, 1986).

\section{O CONTRIBUTO DAS NEUROCIÊNCIAS COGNITIVAS}

As neurociências cognitivas têm vindo a resgatar uma perspetiva biológica, interessada em estudar como é que 0 autoconhecimento se forma, acontece e regula o comportamento a partir do cérebro. Estas novas neurociências juntam, ao estudo dos efeitos das lesões cerebrais (Damásio, 2010; Phillippi et al., 2011), a investigação do cérebro de pessoas saudáveis com as tecnologias de imagiologia funcional (revisão em D’Argembeau, 2015).

Muita investigação tem tentado esclarecer como é processada no cérebro a informação relativa ao "eu", em 


\section{AUTOCONHECIMENTO: UMA BREVE REVISÃO NARRATIVA}

particular se o seu processamento é distinto do processamento da informação relativa a outras pessoas ou objetos. A hipótese de se tratar de uma tarefa especial (e.g., realizada numa área exclusiva do cérebro) tem estado subjacente, dadas as evidências laboratoriais de um certo privilégio no acesso à informação sobre o "eu" e a experiência subjetiva de singularidade e continuidade de cada indivíduo (Guillihan \& Farah, 2005).

Vários estudos têm vindo a convergir na importância do córtex pré-frontal medial no processamento de informação autorreferente (Denny et al., 2012; Guillihan \& Farah, 2005; Legrand \& Ruby, 2009; Northoff et al., 2006) que, no entanto, parece que estar também envolvido no processamento de informação sobre os outros (Denny et al., 2012) e objetos (Legrand \& Ruby, 2009). Denny et al. (2012) confirmaram esta heterogeneidade funcional do córtex pré-frontal medial mas encontraram também um gradiente desde a zona dorsal, mais envolvida na informação sobre outros, até à zona ventral, mais envolvida na informação autorreferente. Parece, pois, plausível que essas regiões do cérebro sejam importantes mas não exclusivas do processamento autorreferencial.

Curiosamente, é o processamento cerebral associado à experiência de subjetividade, continuidade pessoal, etc. que continua a alimentar a hipótese de singularidade do processamento cerebral de informação autorreferente. Tanto Damásio (2010) como Legrand e Ruby (2009) sugerem a possibilidade de esta dimensão subjetiva (a consciência de si mesmo) se formar a partir das representações dos objetos do mundo com que a pessoa contacta, e da representação de como esses objetos modificam a sua experiência motora e sensorial. Entretanto, na literatura sobre a consciência interoceptiva, o sentido de "embodied self" (Mehling et al., 2009) refere-se à medida em que 0 "eu" integra ambos, a mente e 0 corpo.

\section{CONCLUSÕES}

Nas últimas décadas, a investigação do autoconhecimento tem evoluído em novas direções, numa trajetória de conciliação com a complexidade da experiência de si mesmo de cada indivíduo e com a riqueza conceptual dos primeiros autores (James, 1890), trajetória esta possibilitada também pelos avanços das metodologias de investigação e análise de dados. Embora, nesta fase, muitas destas ideias se encontrem dispersas numa literatura muito especializada, é já possível dar sentido à adivinha de Epstein:

What is it that consists of concepts that are hierarchically organized and internally consistent; that assimilates knowledge, yet, itself, is an object of knowledge; that is dynamic, but must maintain a degree of stability; that is unified and differentiated at the same time; that is necessary for solving problems in the real world; and that is subject to sudden collapse, producing total disorganization when this occurs? $(1973$, p. 407).

\section{REFERÊNCIAS BIBLIOGRÁFICAS}

Allport, G. W. (1955). Becoming. Basic considerations for a psychology of personality. New Haven: Yale University Press.

Aron, A., \& McLaughlin-Volpe, T. (2001). Including others in the self. Extensions to own and partner's group membership. In C. Sedikides \& M. B. Brewer (Eds.). Individual Self, Relational Self, Collective Self (pp. 89108). Philadelphia, USA: Psychology Press.

Baumeister, R. F. (1987). How the self became a problem: A psychological review of historical research. Journal of Personality and Social Psychology, 52(1), 163.

Baumeister, R. F., Campbell, J. D., Krueger, J. I. \& Vohs, K. D. (2003). Does high self-esteem cause better performance, interpersonal success, happiness, or healthier lifestyles?. Psychological Science in the Public Interest, 4, 1-44.

Beck, A. T., Rush, A. J., Shaw, B. F. \& Emery, G. (1979). Cognitive Therapy of Depression. New York: Guilford.

Bosma, H. A. \& Kunnen; E. S. (Eds.) (2001). Identity and Emotion: Development Through Self-Organization. Cambridge, UK: Cambridge University Press.

Bracken, B. A. (Ed.) (1996). Handbook of Self-Concept: Developmental, Social, and Clinical Considerations. New York: Wiley. 
Byrne, B. M. (2002). Validating the measurement and structure of self-concept: snapshots of past, present, and future research. American Psychologist, 57(11), 897. Doi:10.1037/0003-066X.57.11.897

Campbell, J. D., Assanand, S. \& Paula, A. D. (2003). The structure of the self concept and its relation to psychological adjustment. Journal of Personality, 71(1), 115-140. D0I: 10.1111/1467-6494.t01-1-00002

Carapeto, M. J. \& Feixas, G. (2019). Self-Knowledge and Depressive Symptoms in Late Adolescence: A Study Using the Repertory Grid Technique. Journal of Constructivist Psychology, 32:1, 81-97. https://doi.org/10.1080/10720537.2018.1433087.

Carapeto, M. J. \& Feixas, G. (2020). The Organization of Self Knowledge in Adolescence: Some Contributions Using the Repertory Grid Technique. European Journal of Investigation in Health, Psychology and Education, 10(1), 408-423. https://doi.org/10.3390/ejihpe10010031

Chen, S., Boucher, H. \& Kraus, M. W. (2011). The relational self. In S. J. Schwartz, K. Luyckx \& V. L. Vignoles (Eds.), Handbook of Identity Theory and Research (pp. 149-175). New York, N.Y.: Springer.

Cross, S. E., Bacon, P. L. \& Morris, M. L. (2000). The relational-interdependent self-construal and relationships. Journal of Personality and Social Psychology, 78, 791-808.

Damásio, A. (2010). O Livro da Consciência: A Construção do Cérebro Consciente. Lisboa: Temas e Debates.

D'Argembeau A. (2015). Self-Knowledge. In A.W. Toga (Ed.), Brain Mapping: An Encyclopedic Reference (vol. 3) (pp. 35-39). Academic Press.

Denny, B. T., Kober, H., Wager, T. D. \& Ochsner, K. N. (2012). A meta-analysis of functional neuroimaging studies of self-and other judgments reveals a spatial gradient for mentalizing in medial prefrontal cortex. Journal of Cognitive Neuroscience, 24(8), 1742-1752.

Epstein, S. (1973). The self-concept revisited. Or a Theory of a Theory. American Psychologist, 28, 404-414.

Erikson, E. (1959). Identity and the Life Cycle. New York: Norton.

Fischer, K. W. \& Ayoub, C. (1994). Affective Splitting and Dissociation in normal and maltreated children: deveIopmental pathways for self in relationships. In D. Cicchetti \& S. L. Toth (Eds), Disorders and dysfunctions of the self. Rochester Symposium on Developmental Psychopathology (pp. 149-222). Rochester, NY: University of Rochester Press.

Fischer, K. W. \& Bidell, T. R. (2006). Dynamic development of action and thought. In W. Damon e R. M. Lerner (Eds.). Theoretical Models of Human Development. Handbook of Child Psychology (6ª Ed., Vol 1, pp. 313399). New York: Wiley.

Gardner, H. \& Davis, K. (2013). The App Generation: How Today's Youth Navigate Identity, Intimacy, and Imagination in a Digital World. New Haven, CT: Yale University Press.

Gergen, K. J. (1991). The Saturated Self: Dilemmas of Identity in Contemporary Life. New York: Basic Books.

Gillihan, S. J. \& Farah, M. J. (2005). Is self special? A critical review of evidence from experimental psychology and cognitive neuroscience. Psychological Bulletin, 131(1), 76.

Harter, S. (2012). The construction of the self: Developmental and sociocultural foundations (2nd Ed.). New York, N.Y.: Guilford.

Hermans, H. J. (2018). Society in the self: A theory of identity in democracy. Oxford University Press.

Higgins, E. T. (1987). Self-discrepancy: A theory relating self and affect. Psychological Review, 94, 319-340.

Higgins, E. T. (1996). The "self digest": self-knowledge serving self-regulatory functions. Journal of Personality and Social Psychology, 71, 1062-1083.

James, W. (1890). The Principles of Psychology. Chicago: Encyclopedia Britannica.

Kashima, Y., Kashima, E., \& Aldridge, J. (2001). Toward cultural dynamics of self-conceptions. In C. Sedikides \& M. B. Brewer (Eds.), Individual Self, Relational Self, Collective Self (pp. 277-298). Philadelphia, USA: Psychology Press.

Kelly, G. A. (1955). The Psychology of Personal Constructs. New York: Norton \& Company.

Lazarus, R. S. (1991). Emotion and adaptation. New York: Oxford University Press. 


\section{AUTOCONHECIMENTO: UMA BREVE REVISÃO NARRATIVA}

Legrand, D. \& Ruby, P. (2009). What is self-specific? Theoretical investigation and critical review of neuroimaging results. Psychological Review, 116(1), 252.

Lichtwarck-Aschoff, A., van Geert, P., Bosma, H. \& Kunnen, S. (2008). Time and identity: A framework for research and theory formation. Developmental Review, 28, 370-400. D0I:10.1016/j.dr.2008.04.001

Linville, P. W. (1987). Self-complexity as a cognitive buffer against stress-related illness and depression. Journal of Personality and Social Psychology, 52, 663-676.

Markus, H. R. (1977). Self-schemata and processing information about the self. Journal of Personality and Social Psychology, 35, 63-78.

Markus, H. R. \& Kitayama, S. (2010). Cultures and selves. A cycle of mutual constitution. Perspectives on Psychological Science, 5(4), 420-430. D0I: 10.1177/1745691610375557

Markus, H. R. \& Kunda, Z. (1986). Stability and malleability of the self-concept. Journal of Personality and Social Psychology, 51, 858-866.

Markus, H. R. \& Nurius, P. (1986). Possible selves. American Psychologist, 41, 954-969.

Mason, T. B., Smith, K. E., Engwall, A., Lass, A., Mead, M., Sorby, M., ... \& Wonderlich, S. (2019). Self-discrepancy theory as a transdiagnostic framework: A meta-analysis of self-discrepancy and psychopathology. Psychological Bulletin, 145(4), 372.

McAdams, D. P. \& McLean, K. C. (2013). Narrative identity. Current Directions in Psychological Science, 22(3), 233-238. DOI: 10.1177/0963721413475622

Mehling, W. E., Gopisetty, V., Daubenmier, J., Price, C. J., Hecht, F. M., \& Stewart, A. (2009). Body awareness: Construct and self-report measures. PLOS ONE, 4, e5614.

Northoff, G., Heinzel, A., de Greck, M., Bermpohl, F., Dobrowolny, H. \& Panksepp, J. (2006). Self-referential processing in our brain —a meta-analysis of imaging studies on the self. Neuroimage, 31(1), 440-457.

Nowak, A., Vallacher, R. R. \& Zochowski, M. (2005). The emergence of personality: Dynamic foundations of individual variation. Developmental Review, 25(3), 351-385. D0l:10.1016/j.dr.2005.10.004

Oosterwegel, A. \& Oppenheimer, L. (1993). The Self-System. Developmental Changes Between and Within SelfConcepts. Hillsdale, NJ: Erlbaum.

Philippi, C. L., Duff, M. C., Denburg, N. L., Tranel, D. \& Rudrauf, D. (2012). Medial PFC damage abolishes the self-reference effect. Journal of Cognitive Neuroscience, 24(2), 475-481.

Rafaeli-Mor, E. \& Steinberg, J. (2002). Self-complexity and well-being: A review and research synthesis. Personality and Social Psychology Review, 6, 31-58.

Rosenberg, M. (1965). Society and the Adolescence Self-Image. Princeton, NJ: Princeton University Press.

Rosenberg, M. (1986). Self-concept from middle childhood through adolescence. In J. Suls \& A. G. Greenwald (Eds.), Psychological Perspectives on the Self (pp. 107-135). Hillsdale, NJ: Earlbaum.

Rosenberg, S. (1997). Multiplicity of selves. In R. D. Ashmore \& L. Jussim, Self and Identity. Fundamental Issues (pp. 23-45). New York: Oxford University Press.

Shavelson, R. J., Hubner, J. J. \& Stanton, G. C. (1976). Self-concept: Validation of construct interpretations. Review of Educational Research, 46, 407-441.

Showers, C. J. \& Zeigler Hill, V. (2007). Compartmentalization and integration: The evaluative organization of contextualized selves. Journal of Personality, 75(6), 1181-1204. D0l: 10.1111/j.1467-6494.2007.00472.x

Van der Meulen, M. (2001). Developments in self-concept theory and research: affect, context and variability. In H. A. Bosma \& E. S. Kunnen (Eds.), Identity and Emotion: Development Through Self-Organization (pp. 1032). Cambridge, UK: Cambridge University Press.

Van Doeselaar, L. V., Becht, A. I., Klimstra, T. A., \& Meeus, W. H. J. (2018). A review and integration of three key components of identity development. European Psychologist, 23(4), 278-288. 